\title{
Biophysical Models of Radiation Bystander Effects: 1. Spatial Effects in Three-Dimensional Tissues
}

\author{
Igor Shuryak, ${ }^{a}$ Rainer K. Sachs ${ }^{b}$ and David J. Brenner ${ }^{a, 1}$ \\ ${ }^{a}$ Center for Radiological Research, Columbia University Medical Center, New York, New York; and ${ }^{b}$ Departments of Mathematics and Physics, \\ University of California Berkeley, California
}

Shuryak, I., Sachs, R. K. and Brenner, D. J. Biophysical Models of Radiation Bystander Effects: 1. Spatial Effects in Three-Dimensional Tissues. Radiat. Res. 168, 741-749 (2007).

Non-targeted (bystander) effects of ionizing radiation are caused by intercellular signaling; they include production of DNA damage and alterations in cell fate (i.e. apoptosis, differentiation, senescence or proliferation). Biophysical models capable of quantifying these effects may improve cancer risk estimation at radiation doses below the epidemiological detection threshold. Understanding the spatial patterns of bystander responses is important, because it provides estimates of how many bystander cells are affected per irradiated cell. In a first approach to modeling of bystander spatial effects in a threedimensional artificial tissue, we assume the following: (1) The bystander phenomenon results from signaling molecules $(S)$ that rapidly propagate from irradiated cells and decrease in concentration (exponentially in the case of planar symmetry) as distance increases. (2) These signals can convert cells to a long-lived epigenetically activated state, e.g. a state of oxidative stress; cells in this state are more prone to DNA damage and behavior alterations than normal and therefore exhibit an increased response $(R)$ for many end points (e.g. apoptosis, differentiation, micronucleation). These assumptions are implemented by a mathematical formalism and computational algorithms. The model adequately describes data on bystander responses in the 3D system using a small number of adjustable parameters. $\odot 2007$ by Radiation Research Society

\section{INTRODUCTION}

There is considerable evidence that ionizing radiation affects cells that are located near the site of irradiation but are not themselves traversed by any particle or photon tracks (1-5). Such non-targeted radiation effects are diverse and cell type specific. They include increased mutagenesis and genomic instability $(6,7)$, differentiation (8), micronucleus formation (9), and either decreased or increased response as regards plating efficiency (10), apoptosis or proliferation $(6,10,11)$. These phenomena, which have

\footnotetext{
${ }^{1}$ Address for correspondence: Center for Radiological Research, Columbia University Medical Center, 630 West 168th St., New York, NY 10032; e-mail: djb3@columbia.edu.
}

been termed radiation-induced bystander effects, were observed not only in cell culture but also in vivo $(12,13)$.

Due to the discovery of bystander effects, it is becoming clear that cells making up an organism respond to ionizing radiation collectively, as parts of large interconnected groups, in addition to individually. Radiation-induced damage to any cell within a group will generate signals that affect many other group members. These bystander signals can induce DNA damage in cells that have not themselves been irradiated and can alter their behavior by perturbing the dynamic equilibrium between becoming committed to proliferation, apoptosis, quiescence or differentiation. The molecular identities of the signals have not yet been determined, but it is known that propagation of bystander signals from cell to cell can involve diffusion through the extracellular medium (14), migration of molecules directly between cells through gap junctions $(4,15-17)$, or both.

The net consequence of bystander phenomena for a multicellular organism may be either amplification or suppression of radiation-induced damage to the organism, because mechanisms favoring both types of responses can be activated by intercellular signaling [e.g. $(18,19)]$. Which mechanisms dominate appears to be highly dependent on the cell type and experimental conditions. For example, in situations where bystander signals induce predominantly differentiation and apoptosis, thereby removing many potentially damaged cells from the clonogenic pool, a net anticarcinogenic effect may occur. Conversely, if bystander-induced mutagenesis is prevalent, a pro-carcinogenic outcome is expected.

The main goal of the present paper is to translate the currently available conceptual understanding of the bystander effect, such as the role of oxidative stress as a likely mediator for this effect $(20,21)$, into a quantitative mathematical model. This task was undertaken previously by several authors, e.g. $(14,22,23)$. Our model focuses specifically on the spatial patterns of the bystander effect. Although describing the details of biochemical signaling pathways is beyond the scope of this model, the formalism is intended to be as mechanistic as possible without undue complexity.

The data set of Belyakov et al. (9) for bystander respons- 
es in an artificial human three-dimensional skin system is modeled. In this experiment, only a thin plane of cells was irradiated with an $\alpha$-particle microbeam, and bystander effect end points (apoptosis and micronucleus yield) were measured in parallel planes located at various distances from the irradiated one. This particular data set was selected because it provides rather detailed information on spatial propagation of the bystander response. In addition, it measures the bystander effect in a tissue surrogate instead of in a monolayer culture and therefore may be a better approximation of processes occurring in vivo.

Quantitative modeling of the spatial aspect of bystander effects is particularly relevant in situations where most cells have only a small probability of suffering any "hits"-as would be the case at low radiation doses and/or low dose rates and/or high LET. Under such conditions, it is important to predict how many bystander cells (i.e. what volume of tissue) will be affected per each cell directly "hit" by an ionizing track. The model developed here can provide such estimates and thus may have important implications for radiation protection, radiotherapy and understanding radiation-induced carcinogenesis at low doses/dose rates (24$31)$.

\section{MODEL HYPOTHESES AND THEIR MATHEMATICAL IMPLEMENTATION}

Based on current data, we propose a plausible sequence of events involved in the bystander effect. Implementing this scenario mathematically involves the following eight specific assumptions and derivation steps:

\section{Molecules Involved in the Bystander Effect}

The bystander effect is produced by radiation-induced signals (referred to here as $S$ ) that travel from irradiated cells to their neighbors and, by molecular mediators, that are induced by these signals in target cells. Recent studies of bystander effects and patterns of cell signaling in general $(10,11,18,32-34)$ suggest that the intercellular signals $(S)$ can be proteins (e.g. cytokines such as TGF- $\beta$ ), small messengers (e.g. cAMP, ATP, IP3), or oxidants such as reactive oxygen and nitrogen species (ROS and RNS). They are released into the medium by irradiated cells and can act in an autocrine, paracrine or long-range endocrine manner, depending on the context and on cell properties (e.g. receptor number on a cell surface) $(18,35-38)$. The smallest molecules (those that are $<1 \mathrm{kDa}$ in molecular weight) can also migrate directly between neighboring cells through gap junctions $(39,40)$.

The net effect of bystander signals on unirradiated cells appears to be rather persistent induced overproduction of ROS and RNS. These oxidant molecules are known to play a regulatory role in many biochemical pathways by redox signaling $(11,20,21,41-45)$. Specifically, they have been implicated in radiation-induced bystander effects and genomic instability $(20,21)$ and in other "non-classical" radiobiological phenomena that are observed in some cell lines [i.e. low-dose hypersensitivity/induced radioresistance (46-48) and the death-inducing effect (49)].

Signal-induced overproduction of oxidants leads to accelerated accumulation of DNA damage and alterations in cell fate, which are the experimentally measured bystander responses (here called $R$ ). In general, a moderate elevation in oxidant concentration induces cells to undergo more rapid turnover (i.e., differentiation and apoptosis of some and compensatory proliferation of others) and migration $(11,50)$. Progressively higher oxidant levels cause mutagenesis, cell senescence, and necrosis [e.g. $(42,43)]$. All of these outcomes are observed as forms of the bystander effect, depending on the cell type and irradiation conditions.

\section{Cell Activation}

The bystander signal (or group of signals) $S$ acts in a probabilistic binary manner by causing cells to switch from an undisturbed background state (no excess oxidant production, low rate of DNA damage accumulation) to an activated bystander effect state (increased oxidant production, elevated rate of DNA damage accumulation). This assumption is based on experimental results suggesting that excess damage in bystander cells is approximately independent of dose over a broad range of radiation doses, i.e. "on or off" in quality [e.g. $(51,52)]$.

At any time $t$ after irradiation, a bystander cell located at distance $x$ from the irradiation site will have a probability of being in the activated state rather than in the background state. This probability, which we denote by $P_{a}$, is described by the following differential equation:

$$
d P_{a} / d t=c_{2} S\left(1-P_{a}\right)-c_{3} P_{a} .
$$

Here $c_{2}$ and $c_{3}$ are rate constants for transition between the two states: $c_{2}$ determines the rate at which the activated state is induced, and $c_{3}$ determines the rate of the opposite process, where activated cells revert back to background. The term $c_{2} S$ represents our assumption that the probability per unit time of converting a cell from the background state to the activated state is proportional to the concentration of the bystander signal $(S)$ at the given time and location. The term $1-P_{a}$ indicates the probability that the given cell has not yet undergone this transition and is therefore available for being activated by the bystander signal. Finally, the term $-c_{3} P_{a}$ indicates that the activated state tends to spontaneously revert to background at a fixed probability per unit time.

\section{Spatial Propagation of Bystander Signals}

Propagation of $S$ in space (i.e. from cell to cell) and induction of the activated state by $S$ occur rapidly, on the scale of a few minutes to a few hours. For example, measurement of production of DNA double-strand breaks in bystander fibroblasts by visualizing $\gamma-\mathrm{H} 2 \mathrm{AX}$ complex formation showed that new complexes are being formed as soon as $2-10$ min after irradiation in cells as distant as $5-7.5 \mathrm{~mm}$ away from irradiated ones (16). These data suggest that the processes of $S$ propagation and $P_{a}$ induction proceed at a speed of $>8 \mu \mathrm{m} / \mathrm{s}$. Other studies [e.g. (53)] found that supra-background $\gamma$-H2AX complexes began to appear in bystander cells somewhat more slowly, $30 \mathrm{~min}$ to a few hours after irradiation.

\section{Accumulation of DNA Damage in Bystander Cells}

Once bystander cells have been activated, they accumulate DNA damage at an abnormally high rate, until they revert to the background state. The return to background (which can be called deactivation) happens relatively slowly, on the scale of many hours to days-much more slowly than initial activation.

These conclusions are supported by measurements of various DNA damage-related end points (e.g. $\gamma-\mathrm{H} 2 \mathrm{AX}$ foci, fractions of apoptotic and micronucleated cells) in the bystander effect context, which indicates that these end points typically do not return to background levels for several days (53). A similar temporal pattern is observed in directly irradiated cells, where these same damage markers remain elevated for days to weeks after exposure to radiation, particularly to high-LET $\alpha$ particles (54). Other phenomena, which may share some common causes with bystander effects, e.g. genomic instability in multiple generations of cultured descendants of irradiated cells $(55,56)$ and persistence of an inflammatory state in animal organs irradiated in vivo (57-59), have an even longer duration.

These observations are consistent with measurements of ROS levels in irradiated cell cultures, which also show that a return to baseline oxidant concentration occurs only many days to weeks after irradiation $(20,56)$. It is likely that elevated ROS levels are sustained by a positive feedback 
loop, where oxidants stimulate their own production, e.g. by damaging mitochondria and inducing genes like $\operatorname{COX} 2(60)$, whose products act in ROS-generating pathways.

Our interpretation of these findings is that DNA damage accumulates more rapidly than normal as long as the activated state (i.e. a state of oxidative stress) is maintained. Although opposing processes (such as DNA repair and removal of dead cells) also operate continually, damage levels cannot return completely to background for at least as long as the cells remain activated and DNA continues to be attacked at an elevated rate.

\section{Temporal Patterns of the Bystander Effect}

The data cited to support assumptions 3 and 4 imply that the processes of bystander signaling (i.e. induction of cells into the activated state) and bystander response (i.e. persistence of cells in the activated state before reversion to the background state) can be thought to operate on different time scales.

After an acute radiation exposure, directly hit cells emit the signal $S$, which quickly propagates outward to bystander cells and begins to activate them. Soon it reaches its maximal spatial range. Most bystander cells that are located within the range of $S$ propagation (and are susceptible to being activated) will be converted to the activated state, and those outside this range will remain mostly unaffected.

The time necessary for these processes to occur, which we call $T$, is, as previously cited experiments suggest, no more than a few minutes or hours long. In contrast, the typical time needed for bystander cells to revert to the background state, i.e. $1 / c_{3}$, where $c_{3}$ is as defined in Eq. (1), is much longer-many hours to several weeks. These differences in time scale between signal propagation/cell activation and persistence of the activated state indicate that in Eq. (1), the term representing the first set of processes, i.e. $c_{2} S\left(1-P_{a}\right)$, is numerically much larger than the term representing the second process, $c_{3} P_{a}$. Therefore, the smaller term $c_{3} P_{a}$ can be neglected, and Eq. 1 can be reduced to the following:

$$
d P_{a} / d t=c_{2} S\left(1-P_{a}\right) .
$$

This approximate formulation reflects the intuitive conclusion that if the damage-inducing activated state is long-lasting, brief delays in its inception caused by kinetics of $S$ propagation/cell activation can be disregarded, as if all activated cells were activated simultaneously.

Equation (2) can be solved to yield the following expression for $P_{a}$ :

$$
P_{a}=1-\exp \left[c_{2} \int_{0}^{T} S(t) d t\right] .
$$

This approximation holds as long as $T \ll 1 / c_{3}$, as experimental data suggest. It shows that $P_{a}$ does not depend on $S$ directly, but is affected only by the time integral of $S$ [i.e. $\int_{0}^{T} S(t) d t$ ], and thus this time integral is what determines the maximal spatial range of $S$.

\section{Mechanism of Bystander Signal Propagation}

To generate an explicit solution for $P_{a}$, the mathematical form of the time integral of $S$ [i.e. $\int_{0}^{T} S(t) d t$ ] needs to be specified. We assume this integral to be an exponentially decreasing function of distance in the case of interest here, where the system effectively has planar symmetry, so that analyzing the gradient involves equations in just one fixed spatial direction $(x)$.

This exponential function is intended to represent spatial kinetics observed in juxtacrine or paracrine signal relay systems, for example calcium wave propagation (61-63). While we do not propose that calcium waves are responsible for the bystander effect, the modes of cell-to-cell signaling operating in calcium wave and bystander processes are probably similar. There appears to be some justification for making this mechanistic analogy, because signal propagation velocities identified by studies of physical injury-induced calcium waves and radiation-induced bystander effects are quite consistent-approximately $10 \mu \mathrm{m} / \mathrm{s}$ (16, 61-65). In ad- dition, calcium fluxes were directly observed in the bystander experiment context in cells exposed to conditioned medium from irradiated cells (10).

In many tissue types, stimulation of one or several cells by agents such as physical injury produces a non-regenerative signaling wave that spreads outward by the "fire-diffuse-fire" mechanism to neighboring cells, decreasing in amplitude over time and distance $(33,34)$ : A stimulated cell "fires", releasing the signal, the signal diffuses locally to reach the adjacent cell through the extracellular medium or through gap junctions, and the adjacent cell responds by "firing" its own burst of signal.

Perhaps the simplest way of describing this type of process mathematically is to assume that each time the signal passes from an activated cell to its immediate neighbor, its intensity is reduced by a constant fraction F. In cases of planar symmetry, the signal intensity $(\mathrm{Fs})$ reaching a cell located $i$ cell diameters away from the initial source of the stimulus is therefore $\mathrm{Fs}=(1-\mathrm{F})^{i}$. If the attenuation factor $\mathrm{F}$ is $>0$, a finite number of cells will be affected (i.e. the signal will have a finite spatial range). If $\mathrm{F}$ approaches 0 , the range will approach infinity. For a signal source in the $(y, z)$ plane (i.e. the plane $x=0)$, this simple function can be rewritten to accommodate distances in micrometers rather than cell diameters: Fs $=\exp \left(-c_{1} x\right)$, where $x$ is distance in micrometers, the adjustable parameter $c_{1}=-\ln (1-\mathrm{F}) / \mathrm{d}$, and $\mathrm{d}$ is the average cell diameter in micrometers.

By combining this expression with our previous assumptions, the following equation for the spatial dependence of the time integral of $S$ is produced:

$$
\int_{0}^{T} S(t) d t=N f(D) \exp \left(-c_{1} x\right)
$$

Here $N$ is the number of irradiated cells located in the plane $x=0$ that are capable of producing $S$. The function $f(D)$ represents the dependence of $S$ on radiation dose/dose rate $(D)$. So far there are insufficient data to specify the shape of this functional dependence, which may, for example, be linear or saturating. For the purposes of this paper, where a data set based on a single-dose acute exposure is used, we treat the function $f(D)$ as a constant. Finally, the parameter $c_{1}$, as defined previously, represents the logarithmic rate at which the time integral of $S$ decreases with distance.

\section{Approximate Explicit Solution for Probability of Cell Activation}

By substituting Eq. (4) into Eq. (3), an explicit solution approximating the probability $\left(P_{a}\right)$ that a bystander cell at distance $x$ away from the irradiated plane will be induced into the activated state can be generated:

$$
P_{a}=1-\exp \left[-c_{2} N f(D) \exp \left(-c_{1} x\right)\right]
$$

\section{Complete Mathematical Form of the Model}

According to our assumptions, while a bystander cell is in the activated state, it accumulates DNA damage at an abnormally high rate. However, the rate of damage production is not the only determinant of observed damage yield that is reported in experimental studies. There are multiple other concurrent processes (e.g. damage repair, cell cycle progression, elimination of apoptotic cells from the tissue). These processes interact with damage production, for example by removing damage (e.g. repair of DNA double-strand breaks) or, alternatively, making the damage observable (e.g. onset of apoptosis after cell cycle checkpoints are reached). Consequently, each type of damage (e.g. $\gamma$-H2AX foci, apoptosis, micronucleation, DNA hypomethylation) has its own temporal kinetics in activated bystander cells (53).

In addition, it is likely that there is a sensitive subpopulation of cells (which we call the susceptible population) in which the bystander response is induced at greater likelihood than in other cells exposed to the same amount of signal (31). This assumption is supported by findings of multiple experiments, where only a few percent of cells exhibit bystander effect end points even at high radiation doses [e.g. $(6,9)]$. The actual 
properties that make cells within this group susceptible to the bystander signal (perhaps position in the cell cycle) are not fully understood and do not affect the structure of our model.

These factors of heterogeneous susceptibility to bystander effects and damage-specific temporal kinetics are beyond the scope of our model, which is concerned primarily with the spatial patterns of bystander responses. We account for the effects of these factors indirectly by assuming that the probability $\left(R_{n}\right)$ that a bystander cell will exhibit a particular damage end point $(n)$ at the time of experimental observation (Tobs) is proportional to the probability that this cell had previously been activated $\left(P_{a}\right)$. Using Eq. (5) for $P_{a}$, this assumption is represented mathematically as follows:

$$
R_{n}=K_{n}\left(1-\exp \left[-c_{2} N f(D) \exp \left(-c_{1} x\right)\right]\right)
$$

Here $K_{n}$ is a damage end point-specific and observation time-specific proportionality constant.

Equation (6) is consistent with the intuitive conclusion that at the time of observation (Tobs), the spatial region where the maximum incidence of the damage of interest (e.g. apoptosis, micronucleation) is found is the same region where the maximum fraction of cells were induced into the activated state by bystander signals.

\section{PARAMETER ESTIMATION}

Equation (6) contains three parameters: $c_{1}, c_{2} N f(D)$, and $K_{n}$. The current level of knowledge about bystander phenomena does not make it feasible to specify the exact nature of bystander signals and the activation process and therefore provide reliable bounds on these parameters from sources independent of the modeled data set. Consequently, parameter values were adjusted to fit the data of Belyakov et al. (9).

The parameter $c_{1}$, which represents the decay rate of the time integral of $S$ with distance away from irradiated cells, can vary over a very wide biologically plausible range, depending on the molecular nature of the signal, cell type and extracellular environment. For example, soluble ligands for the epidermal growth factor receptor (EGFR), which are produced after injury and irradiation, have a mean travel range of $<5$ to tens of micrometers depending on receptor number and rate of endocytosis (38). Injury-induced intercellular calcium waves are not observed at all in some cell types, but in others they propagate over tens to hundreds of micrometers, or even over much larger distances (32, 61-64, 66). Bystander effect studies reveal a similar variability in signal range. In vitro experiments involving confluent cell monolayers covered by a liquid medium suggest essentially infinite propagation distances, where the signal reached the edges of the culture dish hundreds to thousands of micrometers away from the irradiation site in times as short as 10 min (16). However, measurements of bystander effect propagation in three-dimensional tissue-like systems, such as the data set of Belyakov et al. (9) used here, show a finite spatial range of several hundred micrometers. A larger finite range of several millimeters was found in some in vivo studies involving partial-body irradiation of mice (13). Because of this variability, and because the identity of the bystander signals is not specified in the model, we allowed $c_{1}$ to be freely adjusted to the data.
The combination $c_{2} N f(D)$ also plays an important role in the spatial dependence of the bystander response. The number of cells directly hit by radiation $(N)$ could be estimated from the experiment of Belyakov et al. (9): It was equal to 400-800 cells per 8-mm length of the irradiated plane. We chose the mid-range estimate of 600 cells $/ 8 \mathrm{~mm}$, or 75 cells $/ \mathrm{mm}$. All of these cells were assumed to produce $S$. The dose/dose-rate-dependent function $f(D)$, however, cannot be determined from this data set, which involves only a single acute irradiation of $10 \alpha$ particles/cell over $\sim 2 \mathrm{~min}$. Because the value of $f(D)$ is absorbed into the value of the product $c_{2} N f(D)$, for convenience we assigned it to 1.0 concentration $\times \mathrm{h}$. The remaining constant $c_{2}$, with units of (concentration $\times \mathrm{h})^{-1}$, was allowed to be freely adjustable to the data.

The damage/time-specific proportionality constants $K_{n}$, where $n=1$ for apoptosis and 2 for micronucleation, represent the maximum excess fractions of cells expressing these damage end points measured at the observation time Tobs $=72 \mathrm{~h}$. They were adjusted to produce the optimum fit to the data.

In our actual calculations, holding $K_{n} c_{2} N f(D)$ fixed while varying $K_{n}$ and $c_{2} N f(D)$ inversely to each other produced almost no change in the results as long as $K_{n}$ remained within the range consistent with the data. Consequently, the number of adjustable parameters was, to good approximation, 2 instead of 3.

\section{DATA-FITTING PROCEDURE}

The excess (compared with background) fractions of apoptotic and micronucleated cells among bystander cells located at various distances away from the irradiated plane were determined from Belyakov et al. [(9), Fig. 5]. At each section through the tissue model (starting at $200 \mu \mathrm{m}$ and continuing until $1100 \mu \mathrm{m}$ away from irradiated cells, at $100-\mu \mathrm{m}$ intervals), the background apoptosis or micronucleus yields were subtracted from the corresponding experimental data points. Combined error bars were calculated by taking the square root of the sum of squares of error bars for irradiated and background samples.

Best fits to the resulting apoptosis and micronucleation data sets were produced using the approximate solution for $R_{n}$ (Eq. 6). Fitting was accomplished by a customized random-restart simulated annealing algorithm written in the FORTRAN programming language, using 10,000 random initial conditions and 10,000 iterations per initial condition. The function that was minimized to obtain a best fit $(f)$ had the following form:

$$
f=\sum\left[\frac{\mathrm{W}_{1}\left(R_{1}-A\right)^{2}}{d A}+\frac{\mathrm{W}_{2}\left(R_{2}-M\right)^{2}}{d M}\right] .
$$

Here $A$ and $M$ are the measured excess apoptotic and micronucleated cell fractions, $d A$ and $d M$ are their error bars, $R_{1}$ and $R_{2}$ are the approximate solutions given by Eq. (6) using corresponding constants $K_{1}$ and $K_{2}, \mathrm{~W}_{1}$ and $\mathrm{W}_{2}$ are 
TABLE 1

Parameter Fitting Results

\begin{tabular}{|c|c|c|c|c|}
\hline Parameter & Meaning & $\begin{array}{c}\text { Best-fit value } \\
\text { for combined } \\
\text { data sets }\end{array}$ & $\begin{array}{c}\text { Best-fit value } \\
\text { for apoptosis } \\
\text { data only }\end{array}$ & $\begin{array}{l}\text { Best-fit value for } \\
\text { micronucleation } \\
\text { data only }\end{array}$ \\
\hline$c_{1}$ & Spatial decay rate of time integral of $S, \mu \mathrm{m}^{-1}$ & 0.0208 & 0.0122 & 0.00648 \\
\hline $\ln \left[c_{2} N f(D)\right]$ & Natural logarithm of the rate constant for cell activation by $S$ & 19.2 & 11.6 & 4.07 \\
\hline$K_{1}$ & Proportionality constant for bystander signal-induced apoptosis & 0.0283 & 0.0281 & - \\
\hline$K_{2}$ & Proportionality constant for bystander signal-induced micronucleation & 0.00856 & - & 0.0117 \\
\hline
\end{tabular}

the respective weights for the two types of data, and the summation is over all experimental tissue sections from 200 to $1100 \mu \mathrm{m}$ away from the irradiated plane. The weight coefficients were defined as

$$
\mathrm{W}_{1}=\frac{1}{\sum \frac{A}{d A}}, \quad \mathrm{~W}_{2}=\frac{1}{\sum \frac{M}{d M}} .
$$

Fitting was performed for the apoptosis data alone (by setting $\mathrm{W}_{2}=0$ ), for the micronucleation data alone (by setting $\mathrm{W}_{1}=0$ ), and for both data sets combined (by keeping $\mathrm{W}_{1}$ and $\mathrm{W}_{2}$ as defined).

\section{RESULTS}

The best-fit parameter values generated by our algorithm for each of the two data sets and for the combined data are shown in Table 1. Adequate fits were produced in all cases, with the predicted curves intersecting most of the data points/error bars (Figs. 1 and 2).

The best-fit values of parameter $c_{1}$ were $0.0208 \mu \mathrm{m}^{-1}$ for combined data sets, $0.0122 \mu \mathrm{m}^{-1}$ for the apoptosis data alone, and $0.00648 \mu \mathrm{m}^{-1}$ for the micronucleation data alone. Thus signal $(S)$ propagation efficiency should decrease by half at a distance of $\sim 30-110 \mu \mathrm{m}$ and drop to $10 \%$ of maximum at $\sim 110-360 \mu \mathrm{m}$, depending on the data set. Spatial decay rates of this order of magnitude are rea-

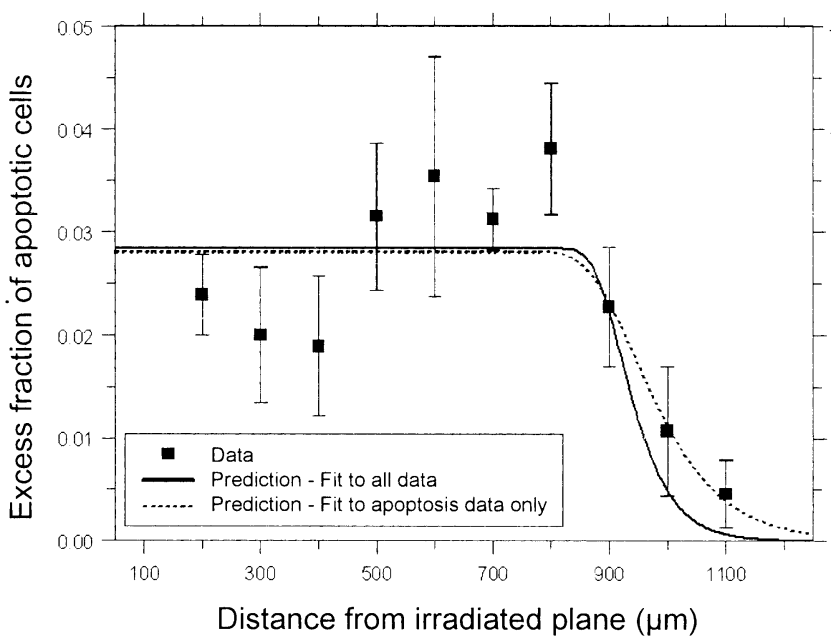

FIG. 1. Measured and predicted excess fractions of apoptotic cells. sonable for juxtacrine/paracrine signaling by small molecules. For example, in keratinocytes, the mean distance to which a calcium wave could be propagated was measured at $90-100 \mu \mathrm{m}(32)$.

Our model fits the data by generating an almost stepfunction-like dependence of $R_{n}$ on distance from the irradiated plane (Figs. 1 and 2). At distances below $\sim 800 \mu \mathrm{m}$ (using the fit for combined data sets as an example), a plateau region is established where essentially all susceptible cells are activated by the bystander signal (i.e. $P_{a} \sim 1$ ). By the time damage is scored (Tobs $=72 \mathrm{~h}$ ), this plateau region exhibits the maximum yield of excess damage end points $\left(R_{n}\right)$. At greater distances (e.g. 1200-1400 $\mu \mathrm{m}$ ), observed excess damage decreases virtually to zero. This putatively occurs because the initial signal $S$ failed to propagate this far away from the irradiated plane and failed to activate the cells present in this more distant region.

From the structure of Eq. (5), it can be deduced that the exponential slope (Slope) with which the bystander activation probability $P_{a}$ decreases at distances beyond the plateau range is linearly dependent on constant $c_{1}$. The extent of the plateau range (Range) is inversely proportional to $c_{1}$ and logarithmically dependent on $c_{2} N f(D)$. Mathematical expressions describing the roles of these parameters are:

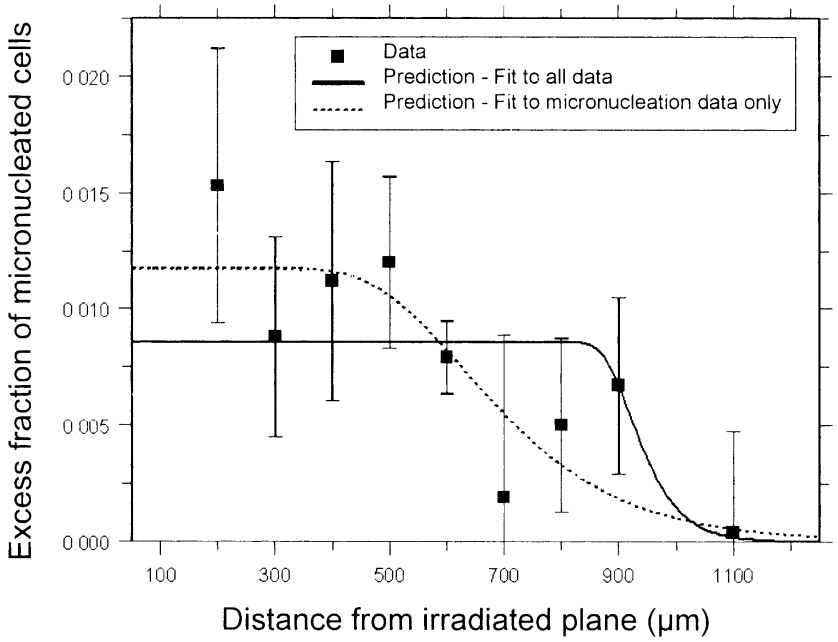

FIG. 2. Measured and predicted excess fractions of micronucleated cells. 
TABLE 2

Calculations of Signal Propagation Efficiency and Range using Best-Fit Parameter Values

\begin{tabular}{|c|c|c|c|c|}
\hline Parameter meaning & Mathematical definition & $\begin{array}{l}\text { Best-fit value } \\
\text { for combined } \\
\text { data sets }\end{array}$ & $\begin{array}{l}\text { Best-fit value } \\
\text { for apoptosis } \\
\text { data only }\end{array}$ & $\begin{array}{l}\text { Best-fit value for } \\
\text { micronucleation } \\
\text { data only }\end{array}$ \\
\hline $\begin{array}{l}\text { Fraction by which } S \text { decreases after traversing } 1 \\
\text { cell diameter }(10 \mu \mathrm{m})\end{array}$ & $\mathrm{F}=1-\exp \left[-10 c_{1}\right]$ & 0.188 & 0.115 & 0.0627 \\
\hline Distance at which $P_{a}$ drops to $0.5, \mu \mathrm{m}$ & $\mathrm{R}_{0.5}=\ln \left[c_{2} N f(D) / \ln (2)\right] / c_{1}$ & 938 & 978 & 685 \\
\hline
\end{tabular}

$$
\begin{aligned}
\text { Slope } & =c_{1}(1-\mathrm{P}) \ln (1-\mathrm{P}) ; \\
\text { Range } & =\ln \left\{c_{2} N f(D) / \ln [1 /(1-\mathrm{P})]\right\} / c_{1} .
\end{aligned}
$$

Here $\mathrm{P}$ is a chosen threshold value. For example, when $\mathrm{P}$ $=0.5$, Range will represent the depth in tissue at which the activation probability (or excess cell damage) drops by half. This range $\left(\mathrm{R}_{0.5}\right)$ is calculated using best-fit parameters for the combined and separate data sets and is shown in Table 2.

Slope can be more conveniently expressed as the fraction (F) by which the bystander signal is reduced from cell to cell, as defined above. For a typical cell diameter of 10 $\mu \mathrm{m}, \mathrm{F}$ is calculated using best-fit parameters for the combined and separate data sets and is also shown in Table 2 .

A pictorial representation of model behavior in response to changes in parameter values is shown in Figs. 3 and 4. When only $c_{1}$ is varied, with $c_{2} N f(D)$ and all other parameters held constant (Fig. 3), the range and slope are both strongly altered. In contrast, when $c_{2} N f(D)$ is varied, leaving everything else unchanged (Fig. 4), the slope is unaffected and the range is modified only slightly. To increase the range significantly, $c_{2} N f(D)$ needs to be increased dramatically (e.g. 10-fold), because range depends on $c_{2} N f(D)$ in a logarithmic manner (Eq. 9). This can be done, for example, by increasing the number of irradiated cells $(N)$ by increasing the thickness of the tissue section exposed to radiation.

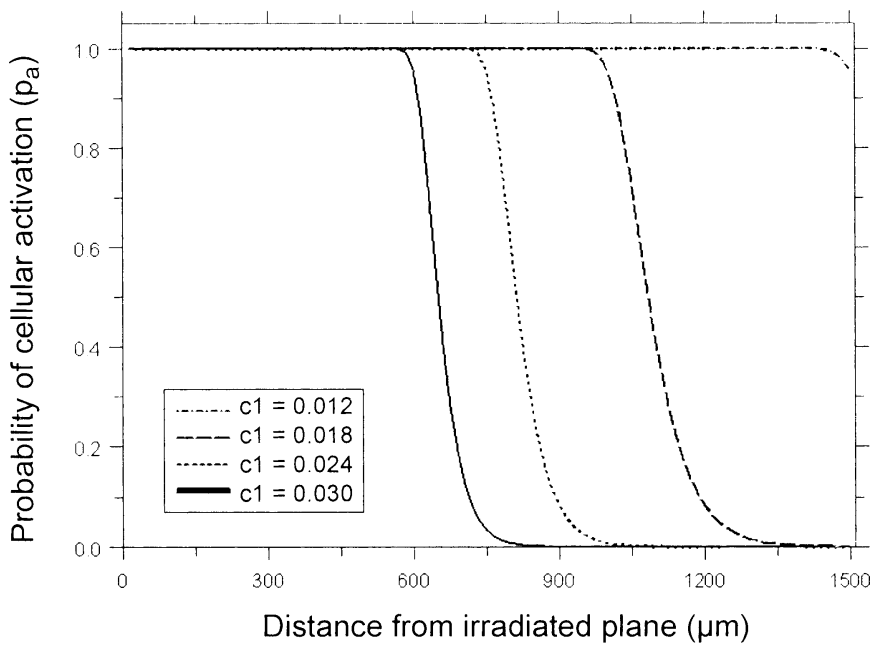

FIG. 3. Probability of cellular activation $\left(P_{a}\right)$ as a function of distance from the irradiated plane $(x$, in $\mu \mathrm{m})$ and parameter $c_{1}$ (in $\left.\mu \mathrm{m}^{-1}\right)$, with all other parameters set to best-fit values for the combined data sets.
As assumed previously (Eq. 6), the bystander response probability $R_{n}$ is proportional to the activation probability $P_{a}$. Consequently, $R_{n}$ has the same step-function-like spatial pattern as $P_{a}$. It is worth noting that whereas the range expression is the same for $R_{n}$ as for $P_{a}$, the slope of $R_{n}$ depends not only on $c_{1}$ but also on $K_{n}$. Thus, to obtain the slope for $R_{n}$, the Slope formula in Eq. (9) needs to be multiplied by $K_{n}$.

The quality of the fit of model predictions to the data depends strongly on $K_{n}$ and Range (i.e. $\mathrm{R}_{0.5}$ ). As is evident from Eq. (9), Range can remain essentially unchanged as long as $\ln \left[c_{2} N f(D)\right]$ and $c_{1}$ are multiplied by a common factor. Consequently, the fit depends substantially on the ratio of $\ln \left[c_{2} N f(D)\right] / c_{1}$ but only weakly on the individual values of these parameters. For example, the best fit for the combined data sets was obtained by $c_{2} N f(D)=2.07 \times 10^{8}$ (i.e. $\left.\ln \left[c_{2} N f(D)\right]=19.2\right)$ and $c_{1}=0.0208 \mu \mathrm{m}^{-1}$, i.e. the ratio of $\ln \left[c_{2} N f(D)\right] / c_{1}=921 \mu \mathrm{m}$ (Table 1). This combination produced $\mathrm{R}_{0.5}=938 \mu \mathrm{m}$ (Table 2). Seemingly very different combinations of $c_{2} N f(D)=5.10 \times 10^{5}$ (i.e. $\left.\ln \left[c_{2} N f(D)\right]=13.1\right)$ with $c_{1}=0.0141 \mu \mathrm{m}^{-1}$, and $c_{2} N f(D)$ $=100$ (i.e. $\ln \left[c_{2} N f(D)\right]=4.61$ ) with $c_{1}=0.00520 \mu \mathrm{m}^{-1}$, in fact generated quite similar $\ln \left[c_{2} N f(D)\right] / c_{1}$ ratios of 932 and $886 \mu \mathrm{m}$, respectively. The values of $\mathrm{R}_{0.5}$ produced by these combinations (958 and $956 \mu \mathrm{m}$ ) were also similar to the best-fitting $\mathrm{R}_{0.5}$.

Therefore, the data sets analyzed here appear to have sufficient power to estimate the bystander signal range, but

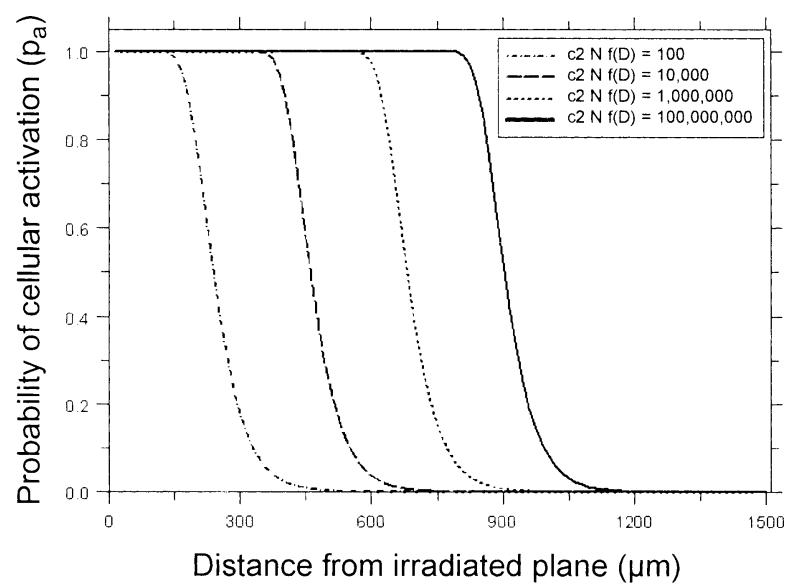

FIG. 4. Probability of cellular activation $\left(P_{a}\right)$ as a function of distance away from the irradiated plane $(x$, in $\mu \mathrm{m})$ and parameter $c_{2} N f(D)$, with all other parameters set to best-fit values for the combined data sets. 
they may not be sufficient to specify the individual values of parameters $c_{1}$ and $c_{2} N f(D)$. For the apoptosis data, the signal range $\left(\mathrm{R}_{0.5}\right)$ is of the order of $950-1000 \mu \mathrm{m}$, and for the micronucleation data it is substantially smaller at 650 $700 \mu \mathrm{m}$. For the combined data sets, $\mathrm{R}_{0.5}$ is apparently dominated by the apoptosis data and is of the order of 930-950 $\mu \mathrm{m}$. The dominance of the apoptosis data set in the simultaneous fit is not an artifact of the fitting procedure, since both apoptosis and micronucleation data sets were weighted equally, with a correction for the values of the data points. Instead, the large value of $\mathrm{R}_{0.5}$ in the combined fit is driven by the high values of the apoptosis data points at distances $500-800 \mu \mathrm{m}$ from the irradiated plane.

The substantial difference in signal range between separate fits to apoptosis and micronucleation data may reflect the possibility that apoptosis and micronucleation are induced by different bystander signals. Until the nature of bystander signals is clarified by further research, the simultaneous model fit to both data sets combined may be useful, because the number of adjustable parameters is reduced, and the fit quality is still adequate for all data.

\section{DISCUSSION}

We have constructed an analytically solvable, simple quantitative model of the spatial and temporal kinetics of the radiation-induced bystander effect. The main assumptions of this model are based on experimental studies suggesting the existence of at least one (and probably many) rapidly spreading signal, which is produced by irradiated cells and induces long-term alterations of the redox balance (activation) in multiple neighboring cells, leading to accumulation of extra DNA damage and modification of cell behavior. The initial signal itself may be oxidative or nonoxidative in nature.

The model describes the selected data sets adequately, using a limited number of parameters. Only two parameter combinations $\left[c_{1}\right.$ and $\left.c_{2} N f(D)\right]$ are required to describe the shape of the bystander response as a function of distance away from the irradiated plane, and the rest act as proportionality constants used for normalization of the data. As new information about the identity of bystander signals and their properties accumulates, increasingly rigorous testing of the model can be performed.

Saturation of the fraction of cells expressing any particular bystander effect end point, which is often observed experimentally, is accounted for by our model. Such saturation could be due to activation of all susceptible cells within the region affected by the bystander signal, followed by expression of the end point by all activated cells. Alternatively, activation may not necessarily lead to expression of the observed end point-a certain probability of expression, which is less than unity, may be involved. In that case, the maximal (saturated) fraction of cells displaying the end point would be the product of three terms: the probability of activation, the susceptible cell fraction, and the proba- bility of expressing the end point after activation of a susceptible cell. Both of these scenarios are consistent with our model.

It is important to note that the model presented here was fitted to a data set generated using an artificial 3D human skin system, which is physiologically much closer to in vivo tissues than are commonly used cell cultures. This realism enhances the potential for predicting the spatial ranges of bystander responses in irradiated human organs.

The ability to quantify these responses is particularly important in situations of low-dose/dose-rate and/or high-LET radiation exposure, where not all cells are traversed by ionizing tracks during the exposure period. Direct experimental measurement of risks (e.g. carcinogenesis) associated with such small doses is not feasible, necessitating the use of mathematical models.

The currently accepted method of carcinogenesis risk estimation at low radiation doses-the linear no-threshold (LNT) approach-involves direct linear extrapolation from higher doses. However, bystander effects are likely to provide a significant pro-carcinogenesis contribution at low radiation doses by increasing the cell turnover and mutation rates, thereby potentially enhancing clonal expansion of pre-existing mutant cells and generating new mutant clones. Such conditions may occur, for example, with cancers induced by radon inhalation (30). The model developed here, which accounts for bystander damage, may enhance current low-dose risk estimation approaches.

\section{ACKNOWLEDGMENTS}

This work was supported by NCI grant 5T32-CA009529 (IS), NASA grant NSCOR04-0014-0017 (RKS), DOE Low Dose Radiation Program grants DE-FG02-03ER63632 and DE-FG02-01ER63226, and NIH grants P41 EB002033-09 and P01 CA-49062 (DJB).

Received: June 4, 2007; accepted: August 28, 2007

\section{REFERENCES}

1. S. A. Lorimore, P. J. Coates and E. G. Wright, Radiation-induced genomic instability and bystander effects: inter-related nontargeted effects of exposure to ionizing radiation. Oncogene 22, 7058-7069 (2003).

2. R. Mikkelsen, Redox signaling mechanisms and radiation-induced bystander effects. Hum. Exp. Toxicol. 23, 75-79 (2004).

3. E. J. Hall, The bystander effect. Health Phys. 85, 31-35 (2003).

4. E. I. Azzam, S. M. de Toledo and J. B. Little, Oxidative metabolism, gap junctions and the ionizing radiation-induced bystander effect. Oncogene 22, 7050-7057 (2003).

5. M. A. Kadhim, S. R. Moore and E. H. Goodwin, Interrelationships amongst radiation-induced genomic instability, bystander effects, and the adaptive response. Mutat. Res. 568, 21-32 (2004).

6. O. V. Belyakov, M. Folkard, C. Mothersill, K. M. Prise and B. D. Michael, A proliferation-dependent bystander effect in primary porcine and human urothelial explants in response to targeted irradiation. Br. J. Cancer 88, 767-774 (2003).

7. T. K. Hei, Cyclooxygenase- 2 as a signaling molecule in radiationinduced bystander effect. Mol. Carcinog. 45, 455-460 (2006).

8. O. V. Belyakov, M. Folkard, C. Mothersill, K. M. Prise and B. D. 
Michael, Bystander-induced differentiation: a major response to targeted irradiation of a urothelial explant model. Mutat. Res. 597, 4349 (2006).

9. O. V. Belyakov, S. A. Mitchell, D. Parikh, G. Randers-Pehrson, S. A. Marino, S. A. Amundson, C. R. Geard and D. J. Brenner, Biological effects in unirradiated human tissue induced by radiation damage up to $1 \mathrm{~mm}$ away. Proc. Natl. Acad. Sci. USA 102, 14203-14208 (2005).

10. F. M. Lyng, P. Maguire, B. McClean, C. Seymour and C. Mothersill, The involvement of calcium and MAP kinase signaling pathways in the production of radiation-induced bystander effects. Radiat. Res. 165, 400-409 (2006)

11. R. Iyer, B. E. Lehnert and R. Svensson, Factors underlying the cell growth-related bystander responses to alpha particles. Cancer Res. 60, 1290-1298 (2000).

12. S. A. Lorimore, J. M. McIlrath, P. J. Coates and E. G. Wright, Chromosomal instability in unirradiated hemopoietic cells resulting from a delayed in vivo bystander effect of gamma radiation. Cancer Res. 65, 5668-5673 (2005).

13. I. Koturbash, R. E. Rugo, C. A. Hendricks, J. Loree, B. Thibault, K. Kutanzi, I. Pogribny, J. C. Yanch, B. P. Engelward and O. Kovalchuk, Irradiation induces DNA damage and modulates epigenetic effectors in distant bystander tissue in vivo. Oncogene 25, 4267-4275 (2006).

14. H. Nikjoo and I. K. Khvostunov, Biophysical model of the radiationinduced bystander effect. Int. J. Radiat. Biol. 79, 43-52 (2003).

15. C. Shao, Y. Furusawa, M. Aoki and K. Ando, Role of gap junctional intercellular communication in radiation-induced bystander effects in human fibroblasts. Radiat. Res. 160, 318-323 (2003).

16. B. Hu, L. Wu, W. Han, L. Zhang, S. Chen, A. Xu, T. K. Hei and Z. $\mathrm{Yu}$, The time and spatial effects of bystander response in mammalian cells induced by low dose radiation. Carcinogenesis 27, 245-251 (2006).

17. G. O. Edwards, S. W. Botchway, G. Hirst, C. W. Wharton, J. K. Chipman and R. A. Meldrum, Gap junction communication dynamics and bystander effects from ultrasoft X-rays. Br. J. Cancer 90, 14501456 (2004).

18. M. H. Barcellos-Hoff and A. L. Brooks, Extracellular signaling through the microenvironment: A hypothesis relating carcinogenesis, bystander effects, and genomic instability. Radiat. Res. 156, 618-627 (2001).

19. K. Baverstock, Radiation-induced genomic instability: a paradigmbreaking phenomenon and its relevance to environmentally induced cancer. Mutat. Res. 454, 89-109 (2000).

20. R. E. Rugo, M. B. Secretan and R. H. Schiestl, X radiation causes a persistent induction of reactive oxygen species and a delayed reinduction of TP53 in normal human diploid fibroblasts. Radiat. Res. 158, 210-219 (2002).

21. C. L. Limoli, E. Giedzinski, W. F. Morgan, S. G. Swarts, G. D. Jones and W. Hyun, Persistent oxidative stress in chromosomally unstable cells. Cancer Res. 63, 3107-3111 (2003).

22. M. P. Little, J. A. Filipe, K. M. Prise, M. Folkard and O. V. Belyakov, A model for radiation-induced bystander effects, with allowance for spatial position and the effects of cell turnover. J. Theor. Biol. 232, 329-338 (2005).

23. F. Ballarini, D. Alloni, A. Facoetti, A. Mairani, R. Nano and A. Ottolenghi, Modelling radiation-induced bystander effect and cellular communication. Radiat. Prot. Dosimetry 122, 244-251 (2006).

24. F. Ballarini, M. Biaggi, A. Ottolenghi and O. Sapora, Cellular communication and bystander effects: a critical review for modelling lowdose radiation action. Mutat. Res. 501, 1-12 (2002).

25. F. Ballarini and A. Ottolenghi, Low-dose radiation action: possible implications of bystander effects and adaptive response. J. Radiol. Prot. 22, A39-A42 (2002).

26. C. Mothersill and C. Seymour, Radiation-induced bystander and other non-targeted effects: novel intervention points in cancer therapy? Curr. Cancer Drug Targets 6, 447-454 (2006).

27. C. Mothersill and C. Seymour, Genomic instability, bystander effects and radiation risks: implications for development of protection strat- egies for man and the environment. Radiats. Biol. Radioecol. 40, 615-620 (2000).

28. C. E. Mothersill, M. J. Moriarty and C. B. Seymour, Radiotherapy and the potential exploitation of bystander effects. Int. J. Radiat. Oncol. Biol. Phys. 58, 575-579 (2004).

29. K. M. Prise, M. Folkard and B. D. Michael, A review of the bystander effect and its implications for low-dose exposure. Radiat. Prot. Dosimetry 104, 347-355 (2003).

30. D. J. Brenner and R. K. Sachs, Domestic radon risks may be dominated by bystander effects - but the risks are unlikely to be greater than we thought. Health Phys. 85, 103-108 (2003).

31. D. J. Brenner, J. B. Little and R. K. Sachs, The bystander effect in radiation oncogenesis: II. A quantitative model. Radiat. Res. 155, 402-408 (2001).

32. S. Koizumi, K. Fujishita, K. Inoue, Y. Shigemoto-Mogami, M. Tsuda and $\mathrm{K}$. Inoue, $\mathrm{Ca}^{2+}$ waves in keratinocytes are transmitted to sensory neurons: the involvement of extracellular ATP and P2Y2 receptor activation. Biochem. J. 380, 329-338 (2004).

33. L. Yang, D. Cranson and V. Trinkaus-Randall, Cellular injury induces activation of MAPK via P2Y receptors. J. Cell Biochem. 91, 938950 (2004).

34. V. E. Klepeis, A. Cornell-Bell and V. Trinkaus-Randall, Growth factors but not gap junctions play a role in injury-induced $\mathrm{Ca}^{2+}$ waves in epithelial cells. J. Cell Sci. 114, 4185-4195 (2001).

35. L. Batsilas, A. M. Berezhkovskii and S. Y. Shvartsman, Stochastic model of autocrine and paracrine signals in cell culture assays. Biophys. J. 85, 3659-3665 (2003).

36. M. Pribyl, C. B. Muratov and S. Y. Shvartsman, Long-range signal transmission in autocrine relays. Biophys. J. 84, 883-896 (2003).

37. S. Y. Shvartsman, M. P. Hagan, A. Yacoub, P. Dent, H. S. Wiley and D. A. Lauffenburger, Autocrine loops with positive feedback enable context-dependent cell signaling. Am. J. Physiol. Cell Physiol. 282, C545-C559 (2002).

38. S. Y. Shvartsman, H. S. Wiley, W. M. Deen and D. A. Lauffenburger, Spatial range of autocrine signaling: modeling and computational analysis. Biophys. J. 81, 1854-1867 (2001).

39. P. A. Weber, H. C. Chang, K. E. Spaeth, J. M. Nitsche and B. J. Nicholson, The permeability of gap junction channels to probes of different size is dependent on connexin composition and permeantpore affinities. Biophys. J. 87, 958-973 (2004).

40. D. V. Krysko, L. Leybaert, P. Vandenabeele and K. D'Herde, Gap junctions and the propagation of cell survival and cell death signals. Apoptosis 10, 459-469 (2005).

41. E. I. Azzam, S. M. De Toledo, D. R. Spitz and J. B. Little, Oxidative metabolism modulates signal transduction and micronucleus formation in bystander cells from alpha-particle-irradiated normal human fibroblast cultures. Cancer Res. 62, 5436-5442 (2002).

42. G. Hughes, M. P. Murphy and E. C. Ledgerwood, Mitochondrial reactive oxygen species regulate the temporal activation of nuclear factor kappaB to modulate tumour necrosis factor-induced apoptosis: evidence from mitochondria-targeted antioxidants. Biochem. J. 389, 83-89 (2005).

43. P. S. Brookes, A. L. Levonen, S. Shiva, P. Sarti and V. M. DarleyUsmar, Mitochondria: regulators of signal transduction by reactive oxygen and nitrogen species. Free Radic. Biol. Med. 33, 755-764 (2002).

44. D. R. Spitz, E. I. Azzam, J. J. Li and D. Gius, Metabolic oxidation/ reduction reactions and cellular responses to ionizing radiation: a unifying concept in stress response biology. Cancer Metastasis Rev. 23, 311-322 (2004).

45. C. Shao, V. Stewart, M. Folkard, B. D. Michael and K. M. Prise, Nitric oxide-mediated signaling in the bystander response of individually targeted glioma cells. Cancer Res. 63, 8437-8442 (2003).

46. C. Mothersill, C. B. Seymour and M. C. Joiner, Relationship between radiation-induced low-dose hypersensitivity and the bystander effect. Radiat. Res. 157, 526-532 (2002).

47. W. M. Bonner, Phenomena leading to cell survival values which deviate from linear-quadratic models. Mutat. Res. 568, 33-39 (2004). 
48. E. Tsoulou, L. Baggio, R. Cherubini and C. A. Kalfas, Low-dose hypersensitivity of V79 cells under exposure to gamma-rays and ${ }^{4} \mathrm{He}$ ions of different energies: survival and chromosome aberrations. Int. J. Radiat. Biol. 77, 1133-1139 (2001).

49. S. Nagar and W. F. Morgan, The death-inducing effect and genomic instability. Radiat. Res. 163, 316-323 (2005)

50. M. Valko, C. J. Rhodes, J. Moncol, M. Izakovic and M. Mazur, Free radicals, metals and antioxidants in oxidative stress-induced cancer. Chem. Biol. Interact. 160, 1-40 (2006).

51. F. M. Lyng, C. B. Seymour and C. Mothersill, Production of a signal by irradiated cells which leads to a response in unirradiated cells characteristic of initiation of apoptosis. Br. J. Cancer 83, 1223-1230 (2000).

52. B. Ponnaiya, G. Jenkins-Baker, D. J. Brenner, E. J. Hall, G. RandersPehrson and C. R. Geard, Biological responses in known bystander cells relative to known microbeam-irradiated cells. Radiat. Res. $\mathbf{1 6 2}$ 426-432 (2004).

53. O. A. Sedelnikova, A. Nakamura, O. Kovalchuk, I. Koturbash, S. A Mitchell, S. A. Marino, D. J. Brenner and W. M. Bonner, DNA double-strand breaks form in bystander cells after microbeam irradiation of three-dimensional human tissue models. Cancer Res. 67, 42954302 (2007).

54. O. V. Belyakov, K. M. Prise, K. R. Trott and B. D. Michael, Delayed lethality, apoptosis and micronucleus formation in human fibroblasts irradiated with X-rays or alpha-particles. Int. J. Radiat. Biol. 75, 985 993 (1999).

55. C. Mothersill, M. A. Kadhim, S. O'Reilly, D. Papworth, S. J. Marsden, C. B. Seymour and E. G. Wright, Dose- and time-response relationships for lethal mutations and chromosomal instability induced by ionizing radiation in an immortalized human keratinocyte cell line. Int. J. Radiat. Biol. 76, 799-806 (2000).

56. H. Tominaga, S. Kodama, N. Matsuda, K. Suzuki and M. Watanabe, Involvement of reactive oxygen species (ROS) in the induction of genetic instability by radiation. J. Radiat. Res. (Tokyo) 45, 181-188 (2004).

57. S. A. Lorimore, P. J. Coates, G. E. Scobie, G. Milne and E. G. Wright, Inflammatory-type responses after exposure to ionizing radiation in vivo: a mechanism for radiation-induced bystander effects? Oncogene 20, 7085-7095 (2001).

58. M. Monleau, M. De Meo, F. Paquet, V. Chazel, G. Dumenil and M. Donnadieu-Claraz, Genotoxic and inflammatory effects of depleted uranium particles inhaled by rats. Toxicol. Sci. 89, 287-295 (2006).

59. J. W. Denham and M. Hauer-Jensen, The radiotherapeutic injury-a complex 'wound'. Radiother. Oncol. 63, 129-145 (2002).

60. H. Zhou, V. N. Ivanov, J. Gillespie, C. R. Geard, S. A. Amundson, D. J. Brenner, Z. Yu, H. B. Lieberman and T. K. Hei, Mechanism of radiation-induced bystander effect: role of the cyclooxygenase-2 signaling pathway. Proc. Natl. Acad. Sci. USA 102, 14641-14646 (2005)

61. S. Coombes, R. Hinch and Y. Timofeeva, Receptors, sparks and waves in a fire-diffuse-fire framework for calcium release. Prog. Biophys. Mol. Biol. 85, 197-216 (2004).

62. S. P. Dawson, J. Keizer and J. E. Pearson, Fire-diffuse-fire model of dynamics of intracellular calcium waves. Proc. Natl. Acad. Sci. USA 96, 6060-6063 (1999).

63. Y. Timofeeva and S. Coombes, Directed percolation in a two-dimensional stochastic fire-diffuse-fire model. Phys. Rev. E Stat. Nonlin. Soft Matter Phys. 70, 062901 (2004).

64. T. Korkiamaki, H. Yla-Outinen, J. Koivunen, S. L. Karvonen and J. Peltonen, Altered calcium-mediated cell signaling in keratinocytes cultured from patients with neurofibromatosis type 1. Am. J. Pathol. 160, 1981-1990 (2002).

65. S. L. Dargan and I. Parker, Buffer kinetics shape the spatiotemporal patterns of IP3-evoked $\mathrm{Ca}^{2+}$ signals. J. Physiol. 553, 775-788 (2003).

66. T. Hofer, A. Politi and R. Heinrich, Intercellular $\mathrm{Ca}^{2+}$ wave propagation through gap-junctional $\mathrm{Ca}^{2+}$ diffusion: a theoretical study. Biophys. J. 80, 75-87 (2001). 